\title{
The foramen of Monro: a review of its anatomy, history, pathology, and surgery
}

\author{
R. Shane Tubbs • Peter Oakes • Ilavarasy S. Maran • \\ Christian Salib • Marios Loukas
}

Received: 26 June 2014 / Accepted: 24 July 2014 / Published online: 31 July 2014

(C) Springer-Verlag Berlin Heidelberg 2014

\begin{abstract}
Introduction The foramen of Monro lies at the junction between the paired lateral ventricles and the third ventricle of the brain. Methods A comprehensive review of the literature was performed focusing on the foramen of Monro.

Conclusions A good understanding of the anatomy of the foramen of Monro is essential for the neurosurgeon, especially with the increasing use of intraventricular endoscopy.
\end{abstract}

Keywords Anatomy · Ventricular system · Brain · CSF . Interventricular foramen

\section{Introduction}

The foramen of Monro is a short conduit between the paired lateral ventricles and the third ventricle of the brain. This deep structure becomes clinically significant when obstructed and leads to obstructive (non-communicating) hydrocephalus. Etiologies of obstruction at the foramen of Monro include infections, congenital atresia, vascular malformations, and neoplastic processes [7]. Herein, we will review the historical discovery of the foramen of Monro, its development, the microsurgical and surface anatomy of the foramen, pathology in the region, and neurosurgical treatments for such pathology.

R. S. Tubbs · P. Oakes · I. S. Maran · C. Salib · M. Loukas

Department of Anatomical Sciences,

St. George's University, St. George's, Grenada

R. S. Tubbs $(\bowtie)$

Pediatric Neurosurgery, Children's of Alabama,

Birmingham, AL, USA

e-mail: shane.tubbs@chsys.org

R. S. Tubbs

Centre of Anatomy and Human Identification,

University of Dundee, Dundee, UK

\section{History}

Alexander Monro secundus (1733-1817) was born into a lineage of Scottish physicians, with his father and son being Alexander Monro primus and tertius, respectively [2]. Together they held the chair of anatomy at the University of Edinburgh for 126 years [17]. Monro secundus recorded detailed descriptions and illustrations regarding the communication between the lateral and third ventricles of the brain as well as describing changes seen in hydrocephalus. Though many credit Monro secundus with the discovery of the interventricular foramen, he stated in his Observations on the structure and function of the nervous system (1783), "These cavities have been described by Galen, and by many succeeding authors of eminence, as all communicating with each other [10]."

Monro secundus is also known for helping to establish the Monro-Kellie hypothesis [9]. The hypothesis states that the sum of volumes of brain parenchyma, CSF, and intracranial blood is constant. These two contributions make him the most well-known of the Monro lineage.

\section{Embryology}

The brain's ventricular system develops from cavities within the neural tube [8]. While the five brain vesicles develop, the cavity in the forebrain divides into the two lateral ventricles and the third ventricle. The lateral ventricles, which form as outpouchings of the rostral third ventricle, are both interconnected with the third ventricle via the foramen of Monro.

During the early stages of hemispheric development, differential growth patterns persist for a considerable period [14]. Formation of the choroidal fissure depends on related growth patterns of surrounding structures. Particularly important is the relatively slow growth of the foramen of Monro, the 
secondary fusion between the lateral diencephalon and medial hemisphere walls encompassing the upper brain stem by the forward growth of the temporal lobe and its pole toward the apex of the orbit, and the massive expansion of the two great cerebral commissures, the fornix and corpus callosum.

At this point, the choroidal fissure is seen as a caudal extension of the interventricular foramen, which arches above the thalamus and a few millimeters from the median plane [14]. Near the caudal end of the thalamus, the foramen of Monro diverges ventrolaterally, with its curve reaching to the tip of the inferior horn of the lateral ventricle. The upper portion of this arch will be overhung by the corpus callosum and throughout its convexity; it is bordered by the fornix and its derivatives [8].

\section{Microsurgical anatomy of the foramen of Monro}

The microsurgical anatomy of the foramen of Monro has been elegantly illustrated and described by Rhoton et al. [18]. The foramen of Monro is located on each side at the junction of the roof and anterior wall of the third ventricle (Figs. 1, 2, 3, 4, 5, and 6). The foramen is bounded anteriorly by the junction of the column and body of the fornix and the anterior pole of the thalamus posteriorly. The shape and size of the foramina correlate with the size of the ventricles: if the ventricles are small, each foramen will be a crescent-shaped opening anteriorly bounded by the concave curve of the fornix and posteriorly by the convex anterior tubercle of the thalamus. As the ventricles enlarge, the foramen on each side becomes more rounded. Structures passing through the foramen include the choroid plexus, the distal branches of the medial posterior

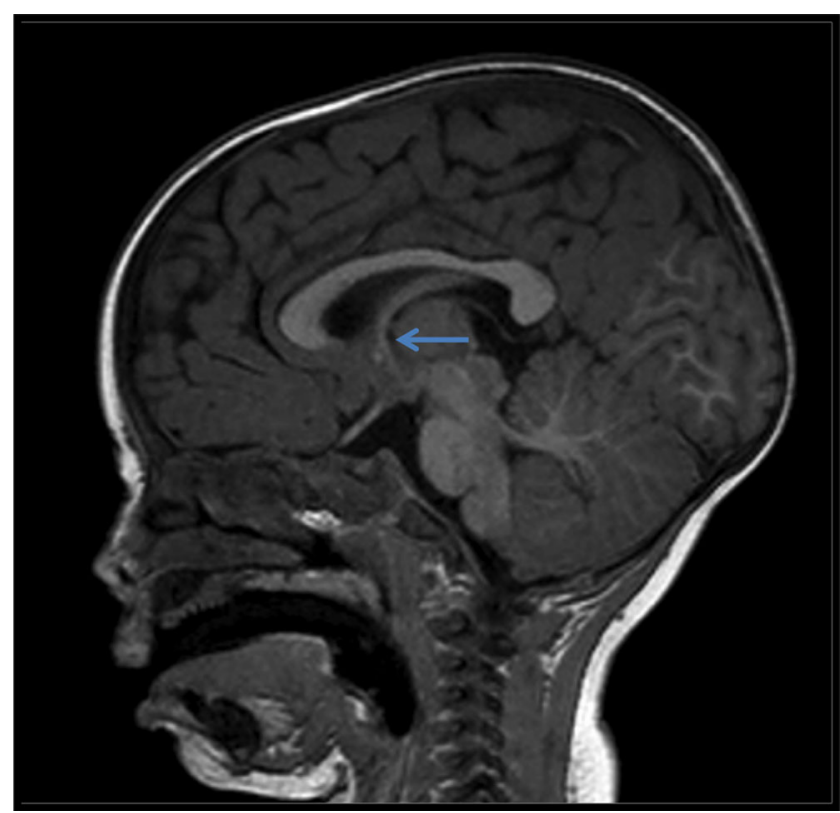

Fig. 2 Midsagittal T1-weighted MRI of the brain noting the right foramen of Monro (arrow)

choroidal arteries, and the, thalamostriate, superior choroidal and septal veins.

\section{Superficial landmarks for the foramen of Monro}

Superficial relationships of a deep landmark are helpful in planning deep operative approaches [13]. At the cranial surface, the foramen of Monro is roughly $2 \mathrm{~cm}$ superior to the pterion, just behind the lower third of the coronal suture. At the cerebral surface, the foramen is located deep to the central
Fig. 1 Midsagittal section of a cadaveric brain illustrating the right foramen of Monro (arrow)

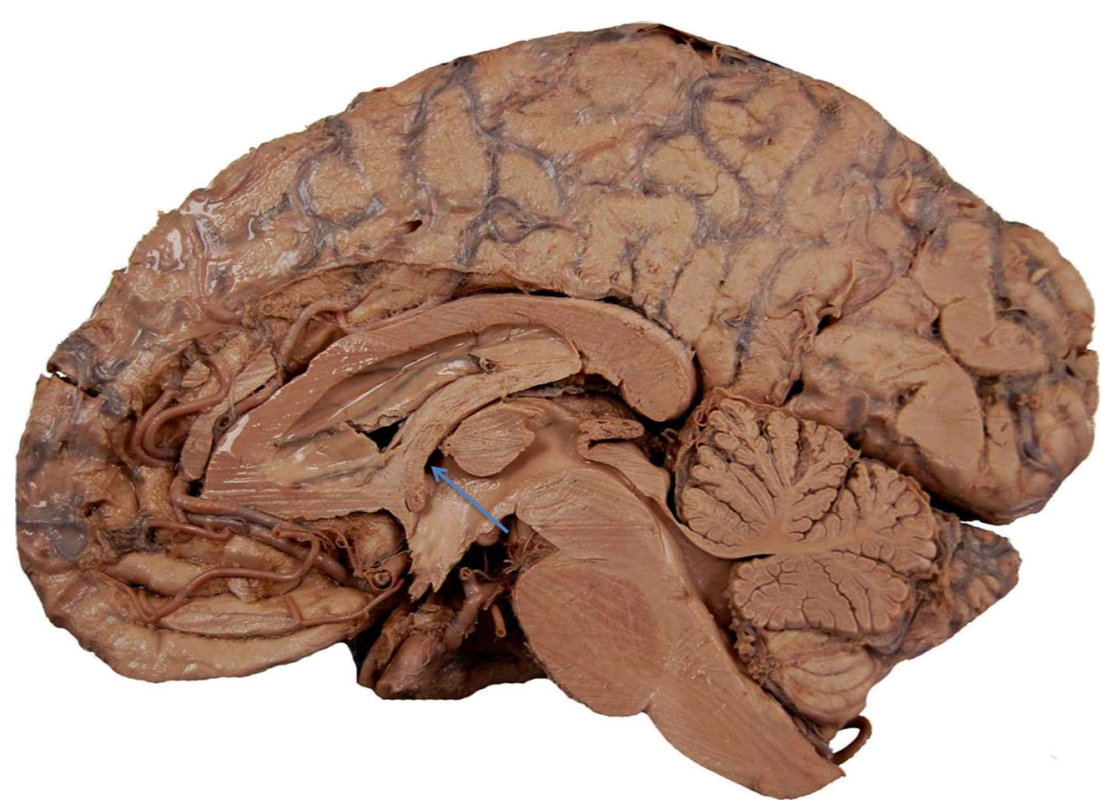




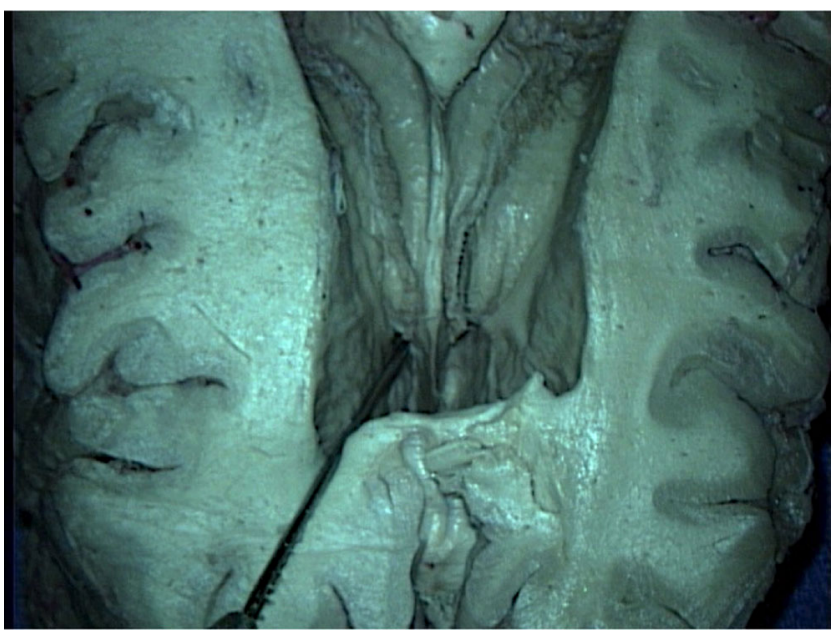

Fig. 3 Superior view of an axial section through a cadaveric brain noting the right foramen of Monro (tip of needle)

portion of the pars opercularis. At the insular level, it is located deep to the central part of the second short insular gyrus. The thalamus is located at the center of the brain with the foramen of Monro at one end and the pineal gland at the other. Together, the surface landmarks of the foramen of Monro and pineal gland estimate the deep position of both the thalamus and third ventricle. The foramen of Monro approximates the anterosuperior thalamic margin, and the pineal gland defines its posterior edge.

\section{Pathology}

The foramen of Monro becomes clinically significant when it is obstructed causing obstructive (non-communicating) hydrocephalus [8]. Stenosis of the foramen of Monro has been attributed to infectious origins (particularly TORCH infections) causing inflammation and scarring in the region, congenital atresia, vascular malformations, and neoplastic processes [7]. Some of the masses most frequently encountered within the

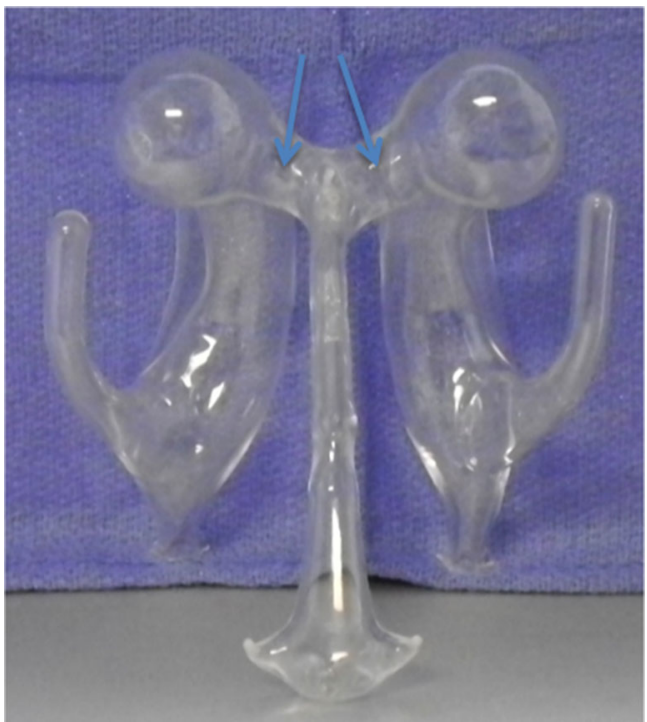

Fig. 5 Glass model of the ventricular system noting the left and right foramina of Monro (arrows)

foramen of Monro include colloid cysts, subependymal giant cell tumors (SGCT) associated with tuberous sclerosis complex (TSC), and subependymal nodules and hamartomas [4].

\section{Colloid cysts}

Colloid cysts are the most common masses of the foramen of Monro [4]. They have an incidence of $0.2-2 \%$ of all intracranial tumors [5]. This well-defined round cyst occurs in adult patients and may be from several millimeters to $3 \mathrm{~cm}$ in size and also attached to the anterosuperior aspect of the third ventricle $[4,12]$. They possess no intrinsic pathological properties and exert symptoms by acting as inert masses. Ninety-percent of colloid cysts are asymptomatic and stable, while $10 \%$ are reported to enlarge or cause hydrocephalus [4]. Rapid enlargement has been associated with drop attacks, dementia, coma, and death [4, 12].
Fig. 4 Right sided foramen of Monro (arrow) as seen through the third ventricle of a cadaver brain. For reference, note the choroid plexus $(C P)$

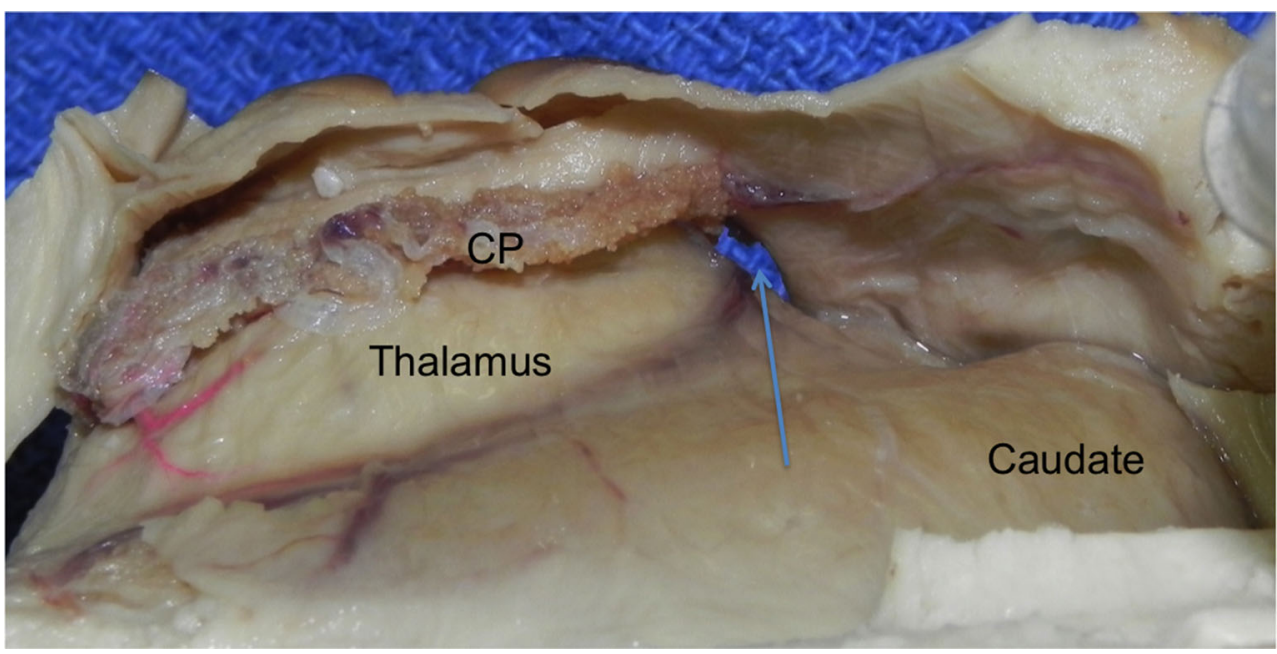




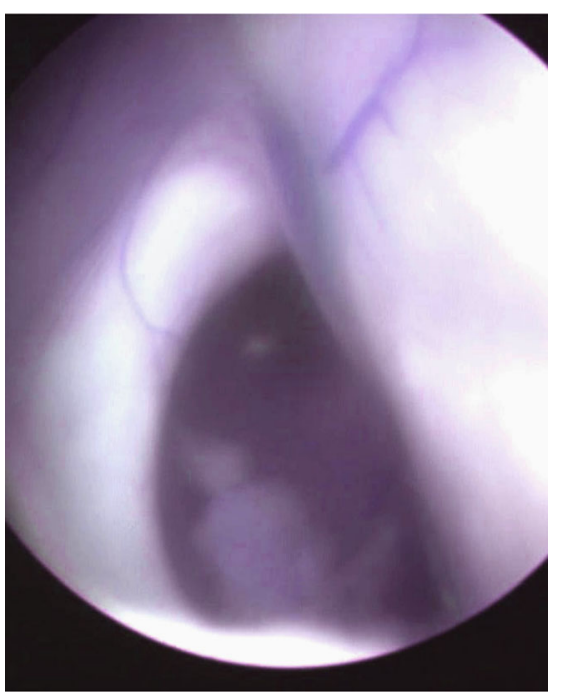

Fig. 6 Endoscopic view of the left foramen of Monro

\section{SGCT associated with TSC}

Subependymal giant cell tumor (SGCT) associated with TSC is the most common pediatric lesion of the foramen of Monro and is found in up to $20 \%$ of patients with TSC [4]. It typically occurs in patients younger than 20-years-old. Subependymal nodules or hamartomas may occur anywhere along the ventricular surface in patients with TSC, but nodules that enlarge over time and which are greater than $1.3 \mathrm{~cm}$ are suspicious for neoplasm. SGCT is a low-grade astrocytoma (WHO grade I). These lesions appear as lobulated, heterogenous, enhancing masses at the foramen of Monro and extend into the lateral or third ventricles.

\section{Subependymoma}

Subependymoma (WHO grade I) is a rare slow-growing tumor most commonly occurring in the fourth ventricle of middle-aged to elderly patients [4]. Supratentorially, it favors the foramen of Monro and appears as a small $(<2 \mathrm{~cm})$, lobulated, well-defined lesion with minimal to moderate enhancement.

\section{Treatment}

Neuroendoscopic intervention

For unilateral membranous obstruction, endoscopic fenestration of the obstructed foramen of Monro is equally as effective as fenestration of the septum pellucidum or foraminoplasty with septostomy [16]. For bilateral membranous obstruction, fenestration of the bilaterally obstructed foramen of Monro can also replace septostomy. When an atretic foramen presents with unclear and unidentifiable borders, foraminoplasty is not recommended as it may injure the column of the fornix and septostomy is preferred [12].

Endoscopically, the thalamostriate vein is located on the posterolateral aspect of the foramen of Monro [16]. During the procedure direct endoscopic vision of the obstructed foramen, careful perforation and fenestration of the slit and thin membrane as well as avoidance of injury to the deep drainage veins surrounding the foramen of Monro are all important for a successful outcome.

Despite being minimally invasive, severe complications, such as hemiparesis and memory deficits can occur [6]. The memory deficit arises from damage to the fornix, a vital link connecting the hippocampus with other structures involved in memory, including the anterior thalami, septal nuclei, and mammillary bodies [11]. The anterior columns of the fornix run close to the foramen of Monro and may be accidentally damaged or deliberately sacrificed during surgical approaches in the region [3]. Such damage leads to persistent anterograde amnesia [15].

\section{Open craniotomy approaches}

There are many surgical approaches for tumors of the ventricular system. Two used in particular to reach the frontal horn of the lateral ventricles and foramen of Monro are the frontal transcortical and anterior transcallosal approaches [1]. In a series of 72 cases, D'Angelo et al. described 21 patients with tumors in the frontal horn of the lateral ventricle or foramen of Monro [1]. Of these, 12 were treated using the transcortical approach and nine via a transcallosal approach.

Transcortical approach-related complications include seizures, hemiparesis, memory loss, confusion, and mutism [19]. Transcallosal approach-related complications include hemiparesis, memory loss, akinetic mutism, and disconnection syndromes. Yaşargil and Abdulrauf [19] stated that based on their experience with a less invasive transcallosal approach (an opening of 10-15 mm), these complications can be avoided.

\section{Conclusions}

Obstruction of the foramen can arise from infectious, congenital, vascular, or neoplastic processes. Treatments include open or endoscopic mass excision or reestablishment of CSF flow. Recently, endoscopic keyhole approaches that emulate the classic craniotomy approaches have been developed. Therefore, a good understanding of the anatomy and relationships of the foramen of Monro are important to the neurosurgeon. 


\section{References}

1. D'Angelo VA, Galarza M, Catapano D, Monte V, Bisceglia M, Carosi I (2008) Lateral ventricle tumors: surgical strategies according to tumor origin and development — a series of 72 cases. Neurosurgery 62(6 Suppl 3):1066-1075

2. Doyle D (2006) Eponymous doctors associated with Edinburgh, Part 2-David Bruce, John Cheyne, William Stokes, Alexander Monro Secundus, Joseph Gamgee. J R Coll Phys Edinb 36:374-381

3. Gaffan EA, Gaffan D, Hodges JR (1991) Amnesia following damage to the left fornix and to other sites. A comparative study. Brain 114: $1297-1313$

4. Glastonbury CM, Osborn AG, Salzman KL (2011) Masses and malformations of the third ventricle: normal anatomic relationships and differential diagnoses. Radiographics 31:1889-1905

5. Hellwig D, Bauer BL, Schulte M, Gatscher S, Riegel T, Bertalanffy H (2008) Neuroendoscopic treatment for colloid cysts of the third ventricle: the experience of a decade. Neurosurgery 62:1101-1109

6. Horn EM, Feiz-Erfan I, Bristol RE, Lekovic GP, Goslar PW, Smith KA, Nakaji P, Spetzler RF (2008) Treatment options for third ventricular colloid cysts: comparison of open microsurgical versus endoscopic resection. Neurosurgery 62:1076-1083

7. Kalhorn SP, Strom RG, Harter DH (2011) Idiopathic bilateral stenosis of the foramina of Monro treated using endoscopic foraminoplasty and septostomy. Neurosurg Focus 30:E5

8. Martin JH (1996) Neuroanatomy: text and atlas. McGraw-Hill, New York
9. Mokri B (2001) The Monro-Kellie hypothesis: applications in CSF volume depletion. Neurology 56:1746-1748

10. Monro A (1783) Observations on the structure and function of the nervous system. Edinburgh

11. Moudgil SS, Azzouz M, Al-Azzaz A, Haut M, Gutmann L (2000) Amnesia due to fornix infarction. Stroke 31:1418-1419

12. Ramamurthi R, Sridhar K, Vasudevan MC (2005) Textbook of operative neurosurgery. BI Publications, New Delhi

13. Rhoton AL Jr (2002) The cerebrum. Neurosurgery 51:S1-S51

14. Standring S (2005) Gray's anatomy: the anatomical basis of clinical practice, 39th edn. Elsevier, New York

15. Vann SD, Denby C, Love S, Montaldi D, Renowden S, Coakham HB (2008) Memory loss resulting from fornix and septal damage: impaired supra-span recall but preserved recognition over a 24 -hour delay. Neuropsychology 22:658-668

16. Wong TT, Lee LS (2000) Membranous occlusion of the foramen of Monro following ventriculoperitoneal shunt insertion: a role for endoscopic foraminoplasty. Childs Nerv Syst 16: 213-217

17. Wu OC, Manjila S, Malakooti N, Cohen AR (2012) The remarkable medical lineage of the Monro family: contributions of Alexander primus, secundus, and tertius. J Neurosurg 116:13371346

18. Yamamoto I, Rhoton AL Jr, Peace DA (1981) Microsurgery of the third ventricle: Part I. Microsurgical anatomy. Neurosurgery 8:334 356

19. Yaşargil MG, Abdulrauf SI (2008) Surgery of intraventricular tumors. Neurosurgery 62:1029-1040 